\title{
Building support for tobacco control
}

\author{
William H Foege
}

This speech was delivered at a plenary session on the closing day of the eighth world conference on tobacco or health in Buenos Aires, Argentina, 30 March-3 April 1992

\section{Introduction}

This week has reinforced our desire for tobacco control while at the same time providing us with examples of solutions that actually work. Yet we know that successful health programmes require more than a demonstrated need and viable solutions. For example, measles may be the most lethal micro-organism the world has ever known. Thirty years after a safe, effective, and inexpensive vaccine became available we are now only beginning to make full use of it. Even more dramatic was the 180 year gap between the first smallpox vaccination by Edward Jenner and global smallpox eradication.

It takes more than a need and the scientific tools to control a health problem. It also takes social will - the willingness to pay the price.

George Bernard Shaw once said that the worst $\sin$ is not to hate people but to be indifferent. That is the essence of inhumanity, and the essence of our challenge...to overcome indifference.

Social will is building for tobacco control, but it is not yet sufficient to effectively counter the strong forces promoting tobacco. Even the group collected in this room will sometimes lose heart.

Sometimes it gets us angry that we need to take the time, to spend the resources, to use the energy to attend a meeting such as this. A meeting that should be totally unnecessary.

Sometimes it gets us angry to see the collective wisdom, talent, and creativity in this

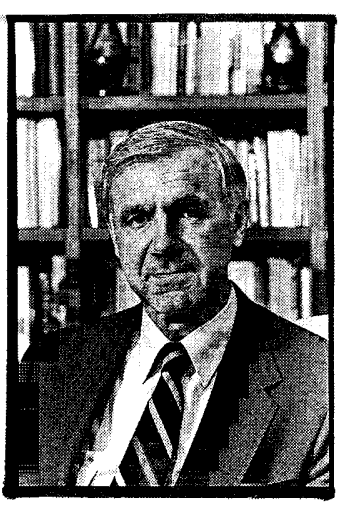

Carter Center of Emory University, One Copenhill, Atlanta, Georgia 30307, USA W H Foege

\section{Core problems}

When we are finished talking at this conference... when the last word has been said about numbers that we can't comprehend... (I can't comprehend 3 million and more deaths a year or even 10000 funerals a day of tobacco victims. But I can comprehend one such funeral for a family member because I have gone through that)...when we have told our final story of loved ones gasping for breath as they lose forever their anticipated retirement to enjoy grandchildren... when we have finished every anecdote...we finally get to the core of our dilemma. We get to the two human conditions that have forced us together this week. ADDICTION and GREED.

What can we do about addiction? We must of course bring all of the support we can muster, to never give up on people, and to provide understanding rather than condemnation. We must focus our anger constructively, rather than blindly, at those caught in this terrible trap. I recently hurt the feelings of a smoker by expressing my exasperation and felt great regret. There is never an excuse for bad manners and we need to recognise that often smokers are as frustrated as we are.

I know the hold of tobacco and am one of the few people I know who actually used to smoke in the shower. To do that requires two things: great addiction and great height. ${ }^{\star}$

To this addiction we need to bring all of the science that is possible. The evidence is convincing. Most people don't smoke because they want to. They smoke because they can't stop. We need to bring science to bear so that stopping can be a free choice.

Third, we need to use what we already know in prevention. When I think of prevention I think of Hugh McCabe, a high school coach, who before dying of lung cancer said, "one of the hardest lessons to teach teenagers is that it is not the last pack of cigarettes that kills, but the first."

Prevention must be the rallying cry of this group, of a caring society, and of an efficient public health strategy.

What can we do about greed? We need to be realistic about what can't be done. We can't neutralise greed. It has been said that avarice is the sphincter of the heart and that it is not

Sometimes we feel overwhelmed at the basic health needs which go unmet because we must respiratory disease, lung cancer, and tobacco induced cardiovascular disease.

Sometimes we can't believe that there really are people willing to make their money by ther people death and suffering. will, it is helpful to ask:

(1) What are the core problems?

(2) What are our core objectives?

(3) What are some of our priority interventions?

(4) What more should we do at this point? 


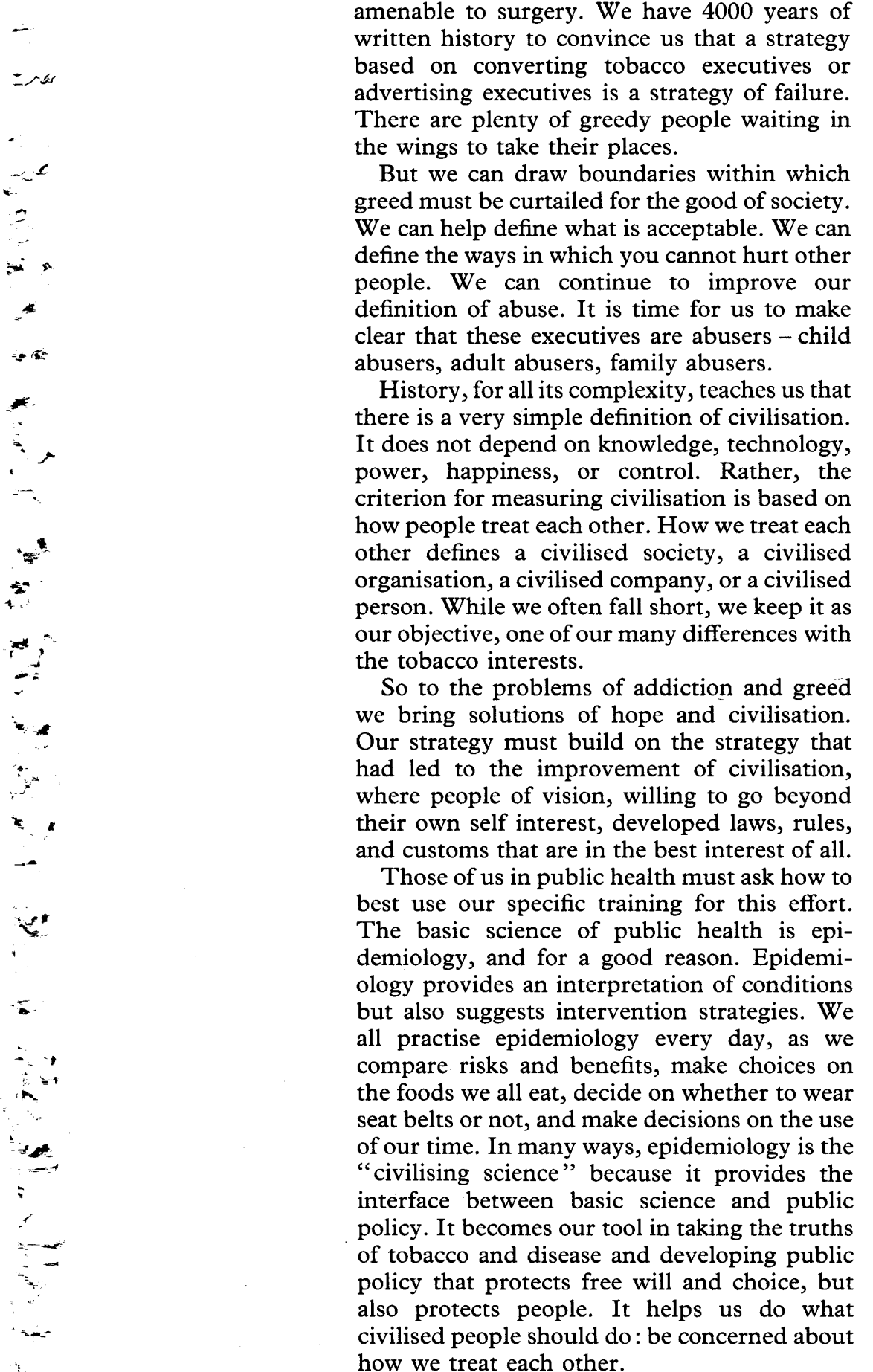

$\div$

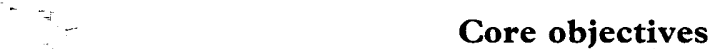

7.

$-$

$=$

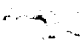

$+\cdots$

t.

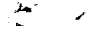

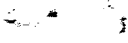

a

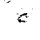

amenable to surgery. We have 4000 years of written history to convince us that a strategy There are plenty of greedy people waiting in But we can draw boundaries within which greed must be curtailed for the good of society. . people. We can continue to improve our do make busers, adult abusers, family abusers. It does not depend on knowledge, technology, power, happiness, or control. Rather, the for measuring civilisation is based on other defines a civilised society, a civilised our objective, one of our many differences with

So to the problems of addiction and greed had led to th where people of vision, willing to go beyond their own self interest, developed laws, rules, best use our specific training for this effort. The basic science of public health is epibut also suggests intervention strategies. We tractise epidemiology every day, as we the foods we all eat, decide on whether to wear "civilising science" because it provides the " policy that protects free will and choice, but civilised people should do: be concerned about how we treat each other.

If our core problems are addiction and greed, what are the objectives we need in order to build social will? They actually are quite simple. We are NOT trying to drive people out of business. We are NOT trying to infringe on free will. We are NOT trying to impose our beliefs.

Our objectives are instead positive. We are attempting to provide everyone with the best quality and quantity of life possible. We are trying to increase freedom, so smoking is truly a choice and not a form of bondage. And we are trying to exercise one of the responsibilities of parenthood, and that is to protect children from harm.

\section{Some key interventions}

We have heard this week about many possibilities and many activities. There is a distinct advantage to multiple projects and simultaneously there are advantages to focusing on some priority interventions. If we understand prevention to be the most effective strategy, then one of our most important interventions has to be a cessation of tobacco advertising.

Of all the things that will confuse historians of the next century, certainly the idea of a lethal product, a product of illness and despair, peddled to youngsters for the profit of the pedlar, will be the most confusing. It would be like now finding that polio was intentionally being spread in the 1950s by the manufacturers of iron lungs.

No single country can effectively stand up to the tobacco companies. Therefore, we should aim at nothing less than a global coalition to stop tobacco advertising. While this should be spearheaded by the World Health Organisation, it must be simultaneously pursued in every country. If Nigeria, for example, bans tobacco advertising, how can they avoid hurting themselves by not having access to international magazines? One solution is that they could ban magazines with tobacco advertising, the law to go into effect when 50 other countries have passed similar laws. Magazines are not going to accept an alternative of stopping distribution in 50 countries at one time. We could help, of course, by developing model legislation to be shared with health ministries around the world.

We have often provided assistance to the tobacco companies by confronting them one state or one country at a time. It is time to mount simultaneous action in every state and in every country. If they have to spread their resources, their legal talent, and their abilities to 100 or 200 hotspots at a time, they are going to lose more encounters.

A second priority prevention intervention is taxation. We have sufficient evidence that taxation alone has value in reducing tobacco demand, but, in addition, we now have experience showing that the combination of taxation and the use of some of the revenue for health education provides a powerful impact on smoking rates. The California experience of doubling the rate of smoking cessation is an example that should be replicated elsewhere. We need to rally public and political support for this demonstrated approach because it appears to be one of the most efficient health promotion strategies we have seen to date.

Third, if prevention is to get its proper place, we need to continue work on school materials that are useful globally. The last decade has seen great strides in effective antitobacco curricula development. Our challenge is replication across cultures.

But most of all, we must promote common sense. Because tobacco is so damaging, people have a right to know what risks they are taking before they start, while they still have free will. Because tobacco is damaging to people in the vicinity of the smoker, these people deserve protection. If people make an informed choice, 
they have that right. But if children make an uninformed choice, or if non-smokers are exposed against their will, tobacco companies should be held responsible. If there is a place for ridicule, it is of the tobacco and advertising executives, who know what they are doing, but are willing, daily, to kill for money. Why do we continue to deal with them as if their actions are socially acceptable?

\section{What more should we do?}

Churchill once advised, "Play for more than you can afford to lose and you will learn the rules of the game." Ten thousand lives a day is more than we can afford to lose so we must learn the rules of the game. What are the rules to implement the above interventions?

First, we must continue to provide factual, scientifically sound information to the public. There has recently been a great deal of publicity over the fact that gunshot deaths now exceed highway deaths in Texas. Most people have no idea that tobacco kills 10 people for every person losing their life on the highways. Why not have local health departments periodically compare tobacco deaths for their city, county, or state with deaths from cars, guns, or other causes that merit media attention?

Sam Levenson once said that it is not hard to be brilliant. He said, think of something very stupid and do the opposite. It is very stupid, ultimately, to tell people they are having fun when they die early, can't enjoy their grandchildren, or suffer the agony of emphysema. How can we be brilliant? By telling the truth. Let's keep repeating the truth with the knowledge that Sheik Rahman was right: "falsehood wins the first battle and truth the last." Let's hear more from those with a diagnosis of lung cancer and hear what they would give for more time with their families or a night with their friends.

To paraphrase the Swiss philosopher Henri Frederic Amiel, truth may be violated by the falsehood of the tobacco companies, but it is outraged by the silence of those who know better, be they scientists or politicians. Kipling reminded us that "words are the most powerful drug used by mankind." Even more powerful than nicotine. We must use them carefully and well.

Second, we need to use and support the medical and public health systems that have been so effective in controlling other health problems.

Alan Blum started something important when he devoted an entire issue of the New York State Fournal of Medicine to the health effects of tobacco. We can now accept it as appropriate that the fournal of the American Medical Association and the American fournal of Public Health do the same thing.

The US Public Health Service has provided almost 30 years of annual Surgeon General's reports on tobacco, and state and local health departments are increasingly involved in tobacco control in ways that are as natural as communicable disease control.

Now the international public health system, under the direction of the World Health
Organisation, is joining the battle. Global health experience is immense but recent. Fifteen years ago the world had never eradicated a disease, and six years ago the world had not reached a majority of children with immunisation. Now it has.

There are similarities between child immunisation and tobacco control which should be exploited. Both require global surveillance systems to measure the problem, intervention effects, and trends. Both need to develop coalitions between health systems and other parts of society, both within and between countries. Both need to effectively mobilise societies to participate in control efforts. Both need to mobilise political support. Both need to promote the idea that we are all in this together-that is, to promote globalism. Einstein once called nationalism an infantile disease, the "measles of mankind." It requires the maturity of globalism to counteract the global problem of tobacco use.

But there are also differences which we need to understand. The enemy in tobacco use is not nature, it is people. The long incubation period from first exposure to disease requires a new way of thinking. There are profits in opposition to tobacco control, for both individuals and governments, which makes this more complicated than measles control.

The public health community should hold tobacco companies to the same rules we require of other companies. In the United States parents cannot have their child immunised in a public clinic without signing a form indicating they understand all of the possible adverse effects of that product. Shouldn't we make sure that a person is at least that well educated before smoking?

Third, in addition to educating the public and support our proved public health systems, we should effectively use the tremendous power that has developed in the spectrum of grassroots tobacco control groups. Henry Ford once noted that there are two types of people: those who think they can and those who think they can't... and they are both right. We are increasingly becoming groups who think they can. These groups are complementary to health departments as they promote social mobilisation, effectively mobilise political involvement, and stimulate medical professionals. They need our support as much as we need theirs.

Finally, what is the major lesson of medicine? The major lesson of medicine is that this is not a fatalistic world: it is possible to plan a rational future. We can change what was once regarded as inevitable, such as smallpox, measles, polio, and now even the effects of tobacco.

Every cigarette that is promoted contributes to an irrational future. It is the theft of life from those who never had a chance to make a choice. A theft of life from their families who depended on a full working life. A theft of life from grandchildren who deserved the counsel of a grandparent.

When tobacco companies promote the joys of smoking, it is not just a lie to consumers, although that is bad enough. It is also an 


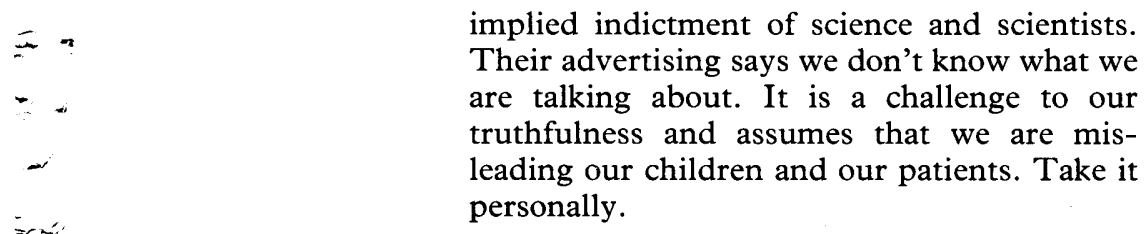

But don't lose heart. In the words of Will Durant, "Civilization is the precarious labor of a minority." Work with the conviction that, even with a minority, it is possible to plan a rational and civilised future. That is our challenge. 